\title{
Haploidentical Family Donor Transplantation for Pediatric Hematologic Malignancies
}

\author{
Jae Wook Lee \\ Division of Hematology and Oncology, Department of Pediatrics, College of Medicine, The Catholic University of Korea, Seoul, Korea
}

\begin{abstract}
Haploidentical family donor hematopoietic stem cell transplantation (Haplo-HSCT) has been increasingly used for patients who require transplant for optimum therapy, but lack a human leukocyte antigen (HLA)-matched donor. Important variables that require consideration in choosing one of potentially many haplo-donors include evaluation for presence of donor-specific anti-HLA antibodies, the age and gender of the donor, the parental relationship of the donor especially for pediatric patients, and $\mathrm{ABO}$ compatibility. Three major platforms have been reported as valid methods of undertaking haplo-HSCT. EX vivo T cell depleted transplant may allow for low rates of graft-versus-host disease, even without pharmacological prophylaxis after transplant. Major impediments such as delayed immune recovery and subsequent infections may be overcome by depletion of specific T cell subsets, as done in $\alpha \beta \mathrm{T}$ cell/B cell depleted transplants. Alternatively, T cell replete haplo-HSCT may be undertaken with post-transplantation cyclophosphamide, or by administering intensive pre- and post-transplant immunosuppression including use of anti-thymocyte globulin. All three haplo-HSCT platforms have been successfully used to treat children with hematologic malignancies. As this method of HSCT is relatively new, long-term follow-up is necessary to improve outcomes and clarify the toxicities of this transplant modality.
\end{abstract}

Key Words: Haploidentical hematopoietic stem cell transplantation, Hematologic malignancy, children, T cell depleted transplantation, Post-transplantation cyclophosphamide, Anti-thymocyte globulin
pISSN 2233-5250 / eISSN 2233-4580 https://doi.org/10.15264/cpho.2021.28.2.67 Clin Pediatr Hematol Oncol 2021;28:67 74

Received on September 27, 2021 Revised on October 7, 2021 Accepted on October 7, 2021
Corresponding Author: Jae Wook Lee Department of Pediatrics, Seoul St. Mary's Hospital, College of Medicine, The Catholic University of Korea, Banpo-daero 222, Seocho-gu, Seoul 06591, Korea Tel: +82-2-2258-6192 Fax: +82-2-537-4544

E-mail: dashwood@catholic.ac.kr ORCID ID: orcid.org/0000-0002-0735-0287

\section{Introduction}

Haploidentical hematopoietic stem cell transplantation (Haplo-HSCT) from a family donor has become an important treatment modality for patients with hematologic malignancies who require transplant, but lack a human leukocyte (HLA) well-matched donor. The number of haplo-HSCTs has continued to increase in recent years. The results of a survey done by the Korean BMT Nurses Society showed that 471 haplo-HSCTs were undertaken in 2019 , comprising $17.4 \%$ of all stem cell trans- plants of that year [1]. Specifically in Korea, difficulties in finding an HLA matched sibling donor (MSD) are likely to increase in the future due to the declining birth rate.

Although haplo-HSCT has gained a significant role in the HSCT field, unresolved issues remain, including selection of the most appropriate donor, the optimum method of T cell depletion, and efficacy of haplo-HSCT compared to more established methods of transplant. In this review, I would like to focus on key aspects of the procedures of haplo-HSCT, and the potential of this mode of transplant in the treatment of children with hematologic malignancies. 


\section{Definition of a Haploidentical Donor}

In essence, a haploidentical donor shares only one of the two HLA loci containing regions of chromosome $6 p$ with the patient. However, the donor-recipient pair may be matched at more than one haplotype. A survey by the European Society for Blood and Marrow Transplantation (EBMT) defined a haploidentical donor as having two or more loci mismatch within HLA-A, -B, -C, -DRB1 andDQB1 loci [2].

\section{Donor Selection}

A patient may potentially have multiple haploidentical family donors, raising the question of transplant from which donor would result in the best HSCT outcome. In deciding this question, multiple issues need to be considered, such as the presence of donor-specific anti-HLA antibodies (DSA) in the patient, donor age and gender, $\mathrm{ABO}$ compatibility and degree of HLA mismatch.

\section{1) Donor-specific anti-HLA antibodies}

One of the first considerations in choosing an appropriate haploidentical donor is to evaluate for DSA in the patient, the incidence of which ranges from 10-20\% [3,4]. Studies have shown that the presence of DSA is significantly linked to delayed hematologic recovery, graft failure and poor graft function after HSCT [3-5]. Also, the level of DSA present in the patient correlates with poor transplant outcome. One study based on 79 adult patients who underwent haplo-HSCT showed that DSA with mean fluorescence intensity $>5,000$ was the only significant risk factor for graft failure [5].

\section{2) Donor age and gender}

Choosing a younger donor over an older donor may decrease the risk of post-transplant clonal hematopoiesis and subsequent secondary malignancy. Younger donors may also experience less difficulty than older donors during the procedures of HSC donation. In a study of 94 pediatric patients with high risk acute leukemia who re- ceived ex vivo T cell depleted (TCD) haplo-HSCT, those who received transplant from a younger donor (with the age threshold set at the median donor age of 40 years) had significantly faster immune reconstitution of all lymphocyte populations post-transplant, and better disease-free survival (DFS) than those who received HSCT from an older donor [6].

Transplants from female donors to male recipients may result in increased risk of graft-versus-host disease (GVHD), as well as graft-versus-leukemia effect, due to donor $\mathrm{T}$ cell mediated response against minor histocompatibility antigens on the patient's Y chromosome [7]. However, within the haplo-HSCT setting, the negative effects of increased GVHD may predominate. Kasamon et al. showed that for patients with hematologic malignancies who received $\mathrm{T}$ cell replete (TCR) haplo-HSCT with high dose post-transplantation cyclophosphamide (PTCy)-based GVHD prophylaxis, a female donor to male patient pairing resulted in significantly worse event-free survival (EFS) in multivariate analysis [8].

The impact of donor gender as an independent factor for HSCT outcome seems to depend upon the haplotransplant platform. For TCD HSCT, Stern et al. showed that patients who received transplant from the mother had significantly better EFS than those who received transplant from the father, due to decreased incidence of both relapse and transplant-related mortality (TRM) [9]. In contrast, a large study based on 990 haplo-HSCTs with PTCy given to adult patients found that a maternal donor resulted in worse progression-free survival (PFS) and overall survival (OS) [10].

Overall, a summary of the literature indicates benefits of selecting a younger donor, and a male donor for a male recipient. For pediatric patients, parents may often be chosen as the haploidentical donor, and in this scenario, the optimum parent donor may depend on the transplant platform; the paternal donor may result in better outcomes for TCR PTCy-based transplant, while the maternal donor may be more appropriate for TCD HSCT, although the scientific basis for the differing effects of donor gender according to each haplo-HSCT platform remain unclear. 


\section{3) Other issues}

A study from the EBMT based on 837 acute myeloid leukemia (AML) patients who received haplo-HSCT reported that major $\mathrm{ABO}$ mismatch was associated with delayed engraftment, while bi-directional mismatch significantly increased the risk of grades II-IV acute GVHD [11]. OS and incidence of relapse and TRM, however, were not affected by $\mathrm{ABO}$ compatibility. Overall, although $\mathrm{ABO}$ compatibility does not seem to affect major transplant outcomes, choosing an ABO-matched donor may contribute to minimizing the complications of HSCT.

The degree of HLA mismatch may differ among potential haploidentical donors, suggesting the possibility of improved transplant outcome for donor-patient pairs with fewer HLA mismatches. However, studies both from PTCy-based haplo-HSCT, as well as from TCR HSCT with intensified GVHD prophylaxis consisting of cyclosporine, mycophenolate mofetil, methotrexate and anti-thymocyte globulin (ATG) show that the degree of HLA mismatch does not influence transplant outcomes [12,13]. Consensus deriving from these data points to forgoing consideration of the degree of HLA mismatch with the patient when choosing among several haploidentical donors.

\section{Haploidentical HSCT Platforms}

1) $\mathrm{T}$ cell depleted HSCT

(1) Initial studies

In 1994, investigators at the University of Perugia published their study on TCD haplo-HSCT in patients with refractory leukemia [14]. The key features of this pivotal study were the attempts to overcome graft failure through an intensified myeloablative conditioning regimen consisting of total body irradiation (TBI), Cy, thiotepa and ATG, and by infusing a 'mega-dose' of stem cells through the combination of bone marrow (BM) and granulocyte colony-stimulating factor (G-CSF) mobilized peripheral blood stem cells (PBSCs). Importantly, no pharmacologic GVHD prophylaxis was given after transplant. Although almost all patients showed donor type engraftment, 9 of 17 patients died of TRM. A subsequent study of 43 patients with high risk acute leukemia reported donor type engraftment in 41 patients, with no instances of GVHD except in one patient who received donor lymphocyte infusion after transplant to aid in immune reconstitution (Fig. 1A) [15]. However, TRM occurred in $40 \%$ of patients, resulting in a low rate of DFS. Although these studies showed that undertaking a TCD HSCT is possible, they also emphasized the increased risk of delayed immune recovery and infectious complications in this mode of transplant.

\section{(2) Pediatric studies}

The feasibility of TCD haplo-HSCT has also been proven in pediatric patients. Lang et al. reported the outcome of 46 children with hematologic malignancies who received myeloablative conditioning-based haplo-transplant [16]. As well as CD3(+) T cells, CD19(+) B cells were depleted ex vivo to decrease the risk of Epstein-Barr virus associated post-transplant lymphoproliferative disease. The study reported primary engraftment in $88 \%$ of patients with low incidences of acute and chronic GVHD. Although TRM of $20 \%$ at 5 years was better than previous studies [14,15], the 3-year EFS of $25 \%$ for the entire cohort of patients remained low.

Among peripheral $\mathrm{T}$ cells, those expressing the $\alpha \beta$ chain ( $\alpha \beta$ T cells) comprise the majority of cells, and are responsible for the cytotoxic pathology of GVHD, whereas $\gamma \delta \mathrm{T}$ cells may aid in suppressing both infection and remnant leukemia post-transplant, with a limited role in initiating GVHD [17]. Bertaina et al. recently reported a comparative study of the outcomes of pediatric patients who received matched unrelated donor (MUD) transplant, mismatched unrelated donor (MMUD) transplant or an $\alpha \beta$ T cell/B cell depleted haplo-transplant [18]. All patients received myeloablative conditioning in first $C R$, and the haplo-transplant recipients received rituximab on day-1. The 98 patients who received $\alpha \beta$ TCD HSCT had significantly lower incidences of both acute and chronic GVHD, and had higher GVHD-free/relapse-free survival (GRFS) than MMUD transplant recipients, emphasizing that $\alpha \beta$ TCD haplo-HSCT is an effective therapeutic option for patients who lack an HLA matched 


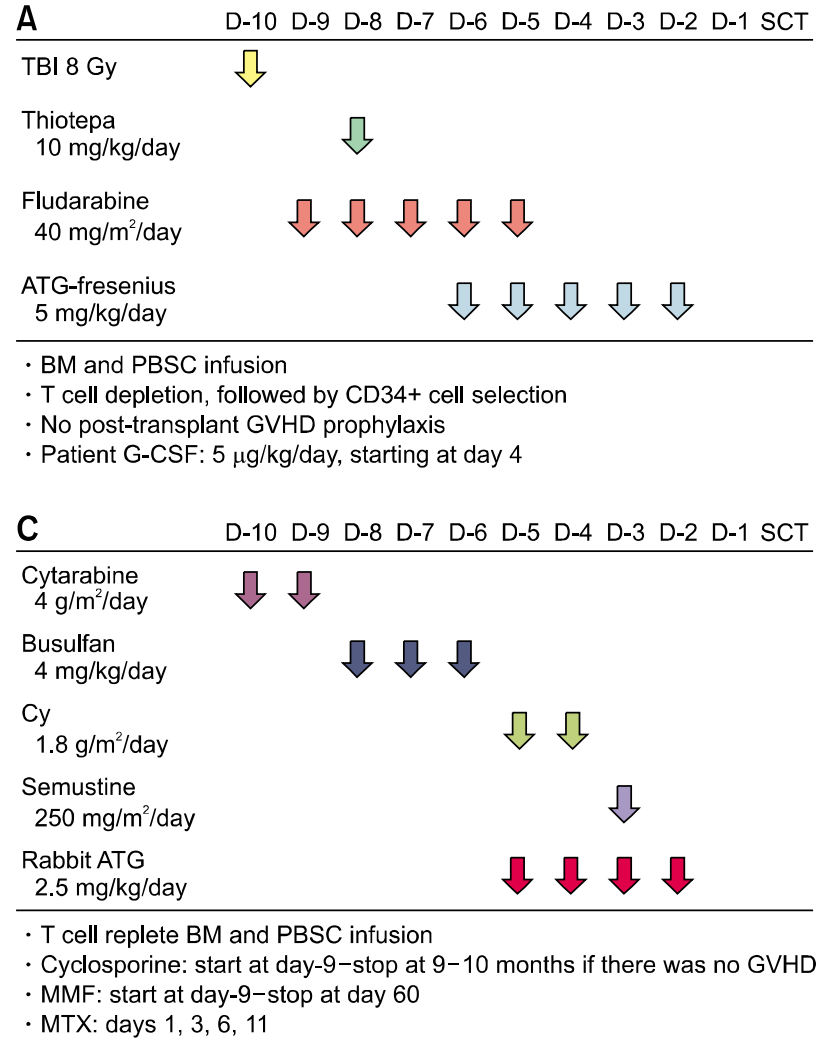

donor. In Korea, Im et al. reported 2-year EFS and OS of $55 \%$ and $65 \%$ for 46 children who received haploHSCT for hematologic malignancies [19]. Receiving transplant with active disease and a history of prior HSCT predicted poor EFS. A subsequent study from the same institution specifically reported the HSCT outcomes of 36 pediatric myelodysplastic syndrome (MDS) patients (12 with advanced MDS), 9 of whom had received TCD haplo-transplant [20]. In contrast to the study by Bertaina et al., 11 of the patients received reduced-intensity conditioning consisting of fludarabine (Flu), reduced dose busulfan $(\mathrm{Bu})$ or $\mathrm{Cy}$, with or without low dose TBI. Rates of OS and TRM were similar between the haplo-transplant and HLA-matched transplant recipients.

\section{2) T cell replete HSCT with post-transplantation cyclophos- phamide}

\section{(1) Initial studies}

In a preclinical animal model of bone marrow transplantation between major histocompatibility complex mismatched donor and recipient, treatment with Cy after

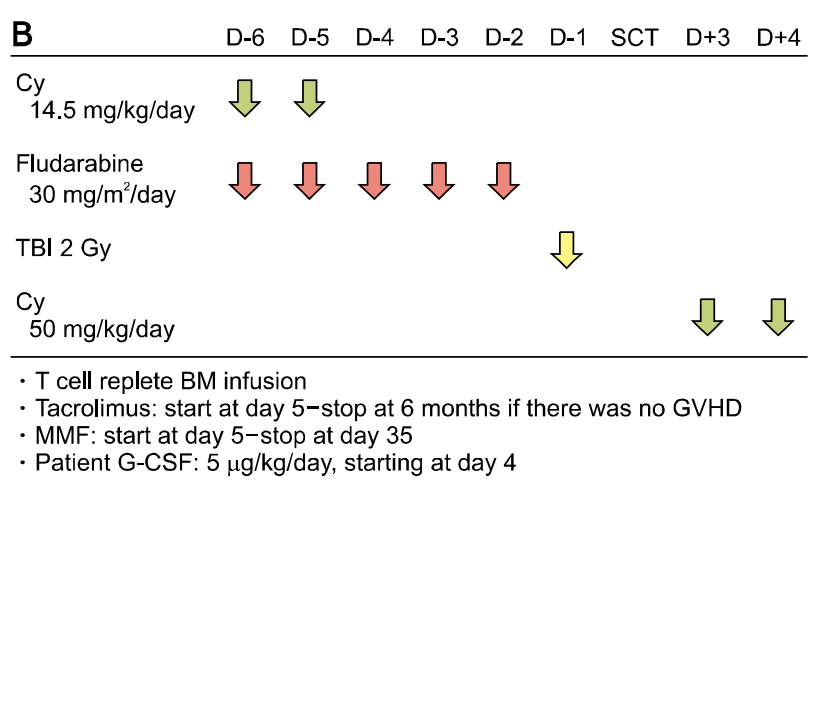

Fig. 1. Examples of conditioning regimens for key methods of haplo-HSCT. (A) TCD HSCT, (B) TCR HSCT with PTCy, (C) TCR HSCT with ATG (GIAC protocol). Note that the doses of ATGFresenius and rabbit ATG are different. TBI, total body irradiation; ATG, antithymocyte globulin; BM, bone marrow; PBSC, peripheral blood stem cells; GVHD, graft-versus-host disease; G-CSF, granulocyte colony-stimulating factor; $\mathrm{Cy}$, cyclophosphamide; MMF, mycophenolate mofetil; MTX, methotrexate.

transplant decreased the severity of acute GVHD by eliminating alloreactive $\mathrm{T}$ cells [21]. Also, hematopoietic progenitor cells express high levels of aldehyde dehydrogenase, conferring resistance of these cells to $\mathrm{Cy}$ in contrast to lymphocytes which have low levels of aldehyde dehydrogenase [22]. Overall, PTCy allows for clearance of the effector cells of GVHD, with limited effect on the precursor cells necessary for rapid donor engraftment.

Based on this concept, investigators at Johns Hopkins University and Fred Hutchinson Cancer Research Center undertook a study of TCR haplo-bone marrow transplantation (BMT) with non-myeloablative conditioning regimen and PTCy given on days 3 and 4 (Fig. 1B) [23]. Sixty-seven patients with advanced hematologic malignancies were treated with this regimen, resulting in limited rates of graft failure and severe acute and chronic GVHD.

\section{(2) Pediatric studies}

Within the framework of haplo-HSCT, a myeloablative conditioning regimen may decrease the incidence of relapse post-transplant. A recent study evaluated 96 chil- 
dren and adults with hematologic malignancies who received standard Bu-based or TBI-based myeloablative conditioning followed by haplo-BMT and PTCy [24]. The cumulative incidence of nonrelapse mortality (NRM) was $11 \%$ at 1 year, and the 3 year EFS was $49 \%$. Although proving the feasibility of this treatment modality, the authors also emphasized the limited number of patients who received myeloablative conditioning-based haplo-transplant in the overall literature, and the need for further follow-up of these patients.

Specifically for pediatric patients, recent studies have focused on a myeloablative conditioning regimen followed by haplo-peripheral blood stem cell transplantation (PBSCT) and PTCy. Jaiswal et al. studied 20 children with acute leukemia treated with a Bu, Flu and melphalan-based conditioning regimen; the 2-year OS was 64\% [25]. In Korea, Hong et al. reported the outcome of 34 children treated with haplo-PBSCT with PTCy, most of whom had malignant disease [26]. The conditioning regimen consisted of $\mathrm{Bu}$, Flu and $\mathrm{Cy}$. The cumulative incidence of grades III-IV acute GVHD and extensive chronic GVHD was 5.9\% and 9.1\% respectively, and the 2 -year EFS for patients with malignant disease was 78\%.

PTCy allows for a convenient method of haplo-HSCT with low risk of GVHD in clinical settings that lack the resources necessary for ex vivo TCD. A question, then, is whether the different haplo-HSCT platforms result in comparable results. Although studies pertaining to children are few, Pérez-Martínez et al. recently compared the outcome of 192 children with hematological malignancies treated with either TCD haplo-HSCT (N=151) or TCR haplo-HSCT with PTCy $(\mathrm{N}=41)$ [27]. Overall, the study found no difference in OS, DFS or relapse incidence between the two haplo-transplant platforms.

\section{3) T cell replete HSCT with anti-thymocyte globulin}

\section{(1) Initial studies}

A final method of haploidentical HSCT, pioneered by investigators at Peking University consists of G-CSF treatment of the donor, intensified immunologic suppression, ATG treatment prior to transplant, and a combination of BM and PBSC infusion (GIAC protocol, Fig.
1C) [28]. The pilot study of this regimen reported on 250 patients with acute leukemia and found that 141 patients survived disease-free, resulting in 3-year leukemia-free survival (LFS) of $71 \%$ for AML and 56\% for acute lymphoblastic leukemia. The incidence of GVHD, however, was significant: $13 \%$ for grades 3-4 acute GVHD and 23\% for extensive chronic GVHD. In Korea, studies based on adult patients with hematological malignancies also showed the efficacy of TCR haplo-PBSCT with ATGbased $\mathrm{T}$ cell depletion, utilizing either a reduced intensity or myeloablative conditioning regimen [29,30].

A key study compared the two TCR haplo-HSCT platforms, PTCy-based ( $\mathrm{N}=193)$ and ATG-based $(\mathrm{N}=115)$, in 308 adult AML patients reported to the EBMT [31]. The study found a significantly lower incidence of grades 3-4 acute GVHD and NRM in patients who received haplo-HSCT with PTCy, although relapse incidence was similar between the two platforms. Overall, patients treated with the PTCy platform had significantly better GRFS and LFS than those treated with ATG-based T cell depletion.

\section{(2) Pediatric studies}

Investigators at Peking University have also shown that ATG-based TCR haplo-HSCT is a valid therapeutic option for pediatric patients. In one large study, $212 \mathrm{pa}^{-}$ tients received haplo-HSCT for acute leukemia with the same ATG-based platform [32]. The incidences of grades 3-4 acute GVHD and extensive chronic GVHD were $14.3 \%$ and $26.6 \%$ respectively. Estimated LFS was similar to a parallel cohort of patients who received MSD HSCT.

\section{Specific Adverse Effects of Haplo-HSCT}

A well-known drawback of TCD haplo-HSCT is delayed immune recovery, although depletion of specific $\mathrm{T}$ cell subsets, such as is done in $\alpha \beta$ TCD HSCT may limit the extent of this complication. Patients may be at particular risk for human herpesvirus-6 (HHV-6) infection. In one study of 38 patients who received either CD34+ cell selection followed by regulatory $\mathrm{T}$ cell (Treg)/conventional $\mathrm{T}$ cell (Tcon) infusion, or CD45RA naïve $\mathrm{T}$ cell depletion, all patients were diagnosed with HHV-6 infection, while none of the patients treated with HSCT 
Table 1. Key characteristics of the 3 haplo-HSCT platoforms

\section{Ex vivo T cell depleted HSCT}

- Potential for very low incidence of acute and chronic GVHD

- No pharmacologic immunosuppression after HSCT

- Higher risk of delayed immune reconstitution and infectious complications, such as HHV-6 infection

- Requirement of equipment and expertise necessary for ex vivo T cell depletion

Post-transplantation cyclophosphamide

- Relatively simple regimen which allows for haplo-HSCT in resource limited settings

- Risk of lower rate of engraftment if undertaken within the context of reduced intensity conditioning

- Possibly a limited role in patients with high susceptibility to alkylating agent toxicity, such as Fanconi anemia patients

- Follow-up is necessary to determine long-term complications of high dose Cy, such as cardiac dysfunction

\section{GIAC protocol}

- Incorporates myeloablative conditioning which may allow for lower incidence of relapse

- Higher incidence of acute and chronic GVHD

- Lengthy period of pharmacologic immunosuppression with multiple agents, which may predispose to increased nonrelapse mortality

with other donor types experienced this infection [33]. Infection occurred early after transplant, at a median of nine days post-transplant in the Treg/Tcon cohort and 13 days post-transplant in the CD45RA depleted cohort. All of the patients responded to antiviral treatment.

For patients who undergo TCR haplo-HSCT with PTCy, high doses of Cy may result in cardiomyopathy, and the risk for this complication may increase in patients with preexisting cardiac dysfunction prior to transplant. Lin et al. evaluated pre- and post-HSCT cardiac function in 176 adult patients who received haplo-HSCT with PTCy [34]. In this cohort, $9.1 \%$ of patients had pre-transplant cardiomyopathy; however, survival after PTCy-based transplant did not differ between patients with or without such preexisting heart complications. Also, although 22\% of the patients developed post-transplant cardiomyopathy, this incidence was similar to that for a cohort of patients who had not received PTCy as part of the transplant. Hence, treatment with PTCy did not act as a major risk factor for the development of cardiomyopathy post-HSCT. That PTCy as a means of GVHD prophylaxis does not predispose towards cardiac toxicities after transplant was further confirmed in a large cohort study of HLA-matched transplant recipients [35]. Considering the much longer potential lifespan after HSCT in pediatric transplant recipients compared with adult patients, further studies are necessary to confirm whether PTCy has limited organ toxicity, including cardiac toxicity, during a long duration of follow-up in patients who received HSCT as children or adolescents.

Finally, the use of $\mathrm{Cy}$ which is an alkylating agent immediately after transplant may lead to clonal changes in the infused HSCs, resulting in donor-derived malignancy (DDM) in the patient. Regarding this potential complication, Majzner et al. evaluated 789 patients who received PTCy and found 4 cases of DDM, resulting in a cumulative incidence of $1.4 \%$ which was similar to previously reported incidence of DDM [36]. Hence, the use of PTCy did not lead to an increase in DDM in patients.

\section{Summary}

The proven efficacy and acceptable safety profile of haplo-HSCT, administered through various transplant platforms, has made HSCT possible for all children regardless of the presence of an HLA-matched donor. For patients with hematological malignancies, haplo-HSCT may play a key role in the treatment of those with high risk leukemias, and may also allow those with relapsed/ refractory disease to undergo rapid transplantation as a component of salvage therapy.

However, further study is required to solve the problems of each haplo-HSCT platform (Table 1). Although TCD haplo-HSCT has been widely studied in the pedia- 
tric population, the cost and infrastructure necessary for ex vivo $\mathrm{T}$ cell depletion remain a barrier for resourcelimited settings. For PTCy, further evaluation seems necessary to clarify possible long-term organ toxicities as pertains to children with much longer post-transplant follow-up. Finally, haplo-HSCT as a mode of therapy remains relatively new compared to transplant utilizing other donors. Long-term, comprehensive study of the pediatric haplo-HSCT patient population will allow for improved HSCT outcomes, while minimizing the complications related to this method of treatment.

\section{Conflict of Interest Statement}

The author has no conflict of interest to declare.

\section{References}

1. Status of blood and marrow transplantation in Korea in 2019. Seoul, Korea: The Korean Society of Blood and Marrow Transplantation, 2021. (Accessed September 9, 2021, at http:// www.bmt.or.kr/main.html)

2. Passweg JR, Baldomero H, Bader P, et al. Use of haploidentical stem cell transplantation continues to increase: the 2015 European Society for Blood and Marrow Transplant activity survey report. Bone Marrow Transplant 2017;52:811-7.

3. Ciurea SO, de Lima M, Cano P, et al. High risk of graft failure in patients with anti-HLA antibodies undergoing haploidentical stem-cell transplantation. Transplantation 2009;88:101924.

4. Chang YJ, Zhao XY, Xu LP, et al. Donor-specific anti-human leukocyte antigen antibodies were associated with primary graft failure after unmanipulated haploidentical blood and marrow transplantation: a prospective study with randomly assigned training and validation sets. J Hematol Oncol 2015; 8:84.

5. Yoshihara S, Maruya E, Taniguchi K, et al. Risk and prevention of graft failure in patients with preexisting donorspecific HLA antibodies undergoing unmanipulated haploidentical SCT. Bone Marrow Transplant 2012;47:508-15.

6. González-Vicent M, Molina B, Deltoro N, et al. Donor age matters in T-cell depleted haploidentical hematopoietic stem cell transplantation in pediatric patients: Faster immune reconstitution using younger donors. Leuk Res 2017;57:60-4.

7. Randolph SS, Gooley TA, Warren EH, Appelbaum FR, Riddell SR. Female donors contribute to a selective graft-versus-leukemia effect in male recipients of HLA-matched, related hematopoietic stem cell transplants. Blood 2004;103:347-52.

8. Kasamon YL, Luznik L, Leffell MS, et al. Nonmyeloablative HLA-haploidentical bone marrow transplantation with high- dose posttransplantation cyclophosphamide: effect of HLA disparity on outcome. Biol Blood Marrow Transplant 2010; $16: 482-9$

9. Stern M, Ruggeri L, Mancusi A, et al. Survival after T cell-depleted haploidentical stem cell transplantation is improved using the mother as donor. Blood 2008;112:2990-5.

10. Mariotti J, Raiola AM, Evangelista A, et al. Impact of donor age and kinship on clinical outcomes after T-cell-replete haploidentical transplantation with PT-Cy. Blood Adv 2020; 4:3900-12.

11. Canaani J, Savani BN, Labopin M, et al. Impact of ABO incompatibility on patients' outcome after haploidentical hematopoietic stem cell transplantation for acute myeloid leukemia - a report from the Acute Leukemia Working Party of the EBMT. Haematologica 2017;102:1066-74.

12. Raiola AM, Risitano A, Sacchi N, et al. Impact of HLA disparity in haploidentical bone marrow transplantation followed by high-dose cyclophosphamide. Biol Blood Marrow Transplant 2018;24:119-26.

13. Huo MR, Pei XY, Li D, et al. Impact of HLA allele mismatch at HLA-A, -B, -C, -DRB1, and -DQB1 on outcomes in haploidentical stem cell transplantation. Bone Marrow Transplant 2018;53:600-8.

14. Aversa F, Tabilio A, Terenzi A, et al. Successful engraftment of T-cell-depleted haploidentical "three-loci" incompatible transplants in leukemia patients by addition of recombinant human granulocyte colony-stimulating factor-mobilized peripheral blood progenitor cells to bone marrow inoculum. Blood 1994;84:3948-55.

15. Aversa F, Tabilio A, Velardi A, et al. Treatment of high-risk acute leukemia with T-cell-depleted stem cells from related donors with one fully mismatched HLA haplotype. N Engl J Med 1998;339:1186-93.

16. Lang P, Teltschik HM, Feuchtinger T, et al. Transplantation of CD3/CD19 depleted allografts from haploidentical family donors in paediatric leukaemia. Br J Haematol 2014;165:68898.

17. Hu Y, Cui Q, Luo C, Luo Y, Shi J, Huang H. A promising sword of tomorrow: Human $\chi \delta$ T cell strategies reconcile allo-HSCT complications. Blood Rev 2016;30:179-88.

18. Bertaina A, Zecca M, Buldini B, et al. Unrelated donor vs HLA-haploidentical $\alpha / \beta$ T-cell- and B-cell-depleted HSCT in children with acute leukemia. Blood 2018;132:2594-607.

19. Im HJ, Koh KN, Seo JJ. Recent advances in haploidentical hematopoietic stem cell transplantation using ex vivo $\mathrm{T}$ cell-depleted graft in children and adolescents. Blood Res 2016;51:8-16.

20. Yoo JW, Im HJ, Kim H, et al. Improved outcomes of allogeneic hematopoietic stem cell transplantation including haploidentical transplantation for childhood myelodysplastic syndrome. Bone Marrow Transplant 2020;55:1595-603.

21. Luznik L, Jalla S, Engstrom LW, Iannone R, Fuchs EJ. Durable engraftment of major histocompatibility complex-incompatible cells after nonmyeloablative conditioning with fludarabine, low-dose total body irradiation, and posttransplantation cyclophosphamide. Blood 2001;98:3456-64.

22. Kastan MB, Schlaffer E, Russo JE, Colvin OM, Civin CI, Hilton 
J. Direct demonstration of elevated aldehyde dehydrogenase in human hematopoietic progenitor cells. Blood 1990;75:194750.

23. Luznik L, O'Donnell PV, Symons HJ, et al. HLA-haploidentical bone marrow transplantation for hematologic malignancies using nonmyeloablative conditioning and high-dose, posttransplantation cyclophosphamide. Biol Blood Marrow Transplant 2008;14:641-50.

24. Symons HJ, Zahurak M, Cao Y, et al. Myeloablative haploidentical BMT with posttransplant cyclophosphamide for hematologic malignancies in children and adults. Blood Adv 2020;4:3913-25.

25. Jaiswal SR, Chakrabarti A, Chatterjee S, et al. Haploidentical peripheral blood stem cell transplantation with post-transplantation cyclophosphamide in children with advanced acute leukemia with fludarabine-, busulfan-, and melphalan-based conditioning. Biol Blood Marrow Transplant 2016; 22:499-504.

26. Hong KT, Kang HJ, Choi JY, et al. Favorable outcome of post-transplantation cyclophosphamide haploidentical peripheral blood stem cell transplantation with targeted busulfan-based myeloablative conditioning using intensive pharmacokinetic monitoring in pediatric patients. Biol Blood Marrow Transplant 2018;24:2239-44.

27. Pérez-Martínez A, Ferreras C, Pascual A, et al. Haploidentical transplantation in high-risk pediatric leukemia: A retrospective comparative analysis on behalf of the Spanish working Group for bone marrow transplantation in children (GETMON) and the Spanish Grupo for hematopoietic transplantation (GETH). Am J Hematol 2020;95:28-37.

28. Huang XJ, Liu DH, Liu KY, et al. Treatment of acute leukemia with unmanipulated HLA-mismatched/haploidentical blood and bone marrow transplantation. Biol Blood Marrow Transplant 2009;15:257-65.

29. Lee $\mathrm{KH}$, Lee JH, Lee JH, et al. Reduced-intensity conditioning therapy with busulfan, fludarabine, and antithymocyte glob- ulin for HLA-haploidentical hematopoietic cell transplantation in acute leukemia and myelodysplastic syndrome. Blood 2011;118:2609-17.

30. Yahng SA, Kim JH, Jeon YW, et al. A well-tolerated regimen of 800 cGy TBI-fludarabine-busulfan-ATG for reliable engraftment after unmanipulated haploidentical peripheral blood stem cell transplantation in adult patients with acute myeloid leukemia. Biol Blood Marrow Transplant 2015;21: 119-29.

31. Ruggeri A, Sun Y, Labopin M, et al. Post-transplant cyclophosphamide versus anti-thymocyte globulin as graft- versus-host disease prophylaxis in haploidentical transplant. Haematologica 2017;102:401-10.

32. Liu DH, Xu LP, Liu KY, et al. Long-term outcomes of unmanipulated haploidentical HSCT for paediatric patients with acute leukaemia. Bone Marrow Transplant 2013;48:1519-24.

33. Perruccio K, Sisinni L, Perez-Martinez A, et al. High incidence of early human herpesvirus -6 infection in children undergoing haploidentical manipulated stem cell transplantation for hematologic malignancies. Biol Blood Marrow Transplant 2018;24:2549-57.

34. Lin CJ, Vader JM, Slade M, DiPersio JF, Westervelt P, Romee R. Cardiomyopathy in patients after posttransplant cyclophosphamide-based hematopoietic cell transplantation. Cancer 2017;123:1800-9.

35. Yeh J, Whited LK, Saliba RM, Rondon G, Popat UR. Cardiac toxicities after HLA-matched allogeneic hematopoietic cell transplantation (Allo-HCT): post-transplant cyclophosphamide (PTCy) is not associated with higher incidence of cardiotoxicity. Biol Blood Marrow Transplant 2020;26(Suppl):S3934 .

36. Majzner RG, Mogri H, Varadhan R, et al. Post-transplantation cyclophosphamide after bone marrow transplantation is not associated with an increased risk of donor-derived malignancy. Biol Blood Marrow Transplant 2017;23:612-7. 